\title{
Effects of a Focused Breathing Mindfulness Exercise on Attention, Memory, and Mood: The Importance of Task Characteristics
}

\author{
Nikolett Eisenbeck, ${ }^{1,2}$ Carmen Luciano' and Sonsoles Valdivia-Salas ${ }^{3}$ \\ ${ }^{\prime}$ Department of Psychology, University of Almería, Almería, Spain \\ ${ }^{2}$ Karoli Gaspar University of the Reformed Church in Hungary \\ ${ }^{3}$ Department of Psychology, University of Zaragoza, Zaragoza, Spain
}

\begin{abstract}
Previous research has shown that long-term mindfulness training has beneficial effects on cognitive functioning and emotional regulation, but results are mixed regarding single mindfulness exercises, especially on attention and memory tasks. Thus, the present study aimed to analyse the effects of the Focused Breathing Exercise (FB) on cognitive performance, using standardised tests. Forty-six healthy undergraduate students were randomly assigned either to a FB or a Control condition. Two cognitive tasks (the Concentrated Attention task of the Toulouse-Pierron Factorial Battery and the Logical Memory Subtest I from the Wechsler Memory Scale III), along with mood evaluations (the Positive and Negative Affect Scale), were implemented both before and after the interventions. Results showed no significant differences for the attention task and mood evaluations. Nonetheless, the FB enhanced performance for the memory task significantly more than the Control exercise. The findings highlight that mindfulness does not affect equally all types of cognitive performances. Task characteristics may be important and their analysis can help to disentangle how mindfulness interferes with cognitive processes.
\end{abstract}

Keywords: mindfulness, cognitive tasks, focused breathing

Mindfulness exercises and mindfulness-based psychotherapies have proven to be successful in enhancing cognitive performance, improving emotional and behavioural regulation, as well as reducing a wide range of psychological symptoms (e.g., Chambers, Lo, \& Allen, 2008; Chiesa, Calati, \& Serretti, 2011; Keng, Smoski, \& Robins, 2011; Khoury et al., 2013; Lykins \& Baer, 2009). Typically, long-term mindfulnessbased interventions such as Mindfulness-Based Stress Reduction (MBSR; Kabat-Zinn, 1990) and Mindfulness-Based Cognitive Therapy (MBCT; Segal, Williams, \&Teasdale, 2002a) comprise therapeutic packages of different techniques and exercises. It is assumed that these sets of mindfulness practices may influence behaviour by targeting several, possibly overlapping processes, such as elevated mindful awareness, defusion, increased attentional control, exposure, and behavioural self-regulation (e.g., Brown, Ryan, \& Creswell, 2007; Khoury et al., 2013; Shapiro, Carlson, Astin, \& Freedman, 2006; Shonin, Van Gordon, \& Griffiths, 2013).

\footnotetext{
Address for correspondence: Dr Nikolett Eisenbeck, Karoli Gaspar University of the Reformed Church in Hungary, Bécsi Street 324, H-1037 Budapest.

Email: eisenbeckniki@gmail.com
}

Behaviour Change | Volume 35 | Number 1| 2018 | pp. 54-70 | C) The Author(s) 2018

doi $10.1017 /$ bec.2018.9 
This scenario has encouraged researchers to conduct short-term, controlled experiments that assess the immediate effects of each exercise and analyse how they affect the participants' behaviour. Typically, the target population has been non-meditators, in order to prevent previous experience from affecting the obtained results. The majority of these short-term, controlled studies implemented a variation of the Focused Breathing exercise (FB), also known as the mindful breathing exercise, or breathing meditation. This exercise is at the core of a number of traditional meditation practices and is usually the first to be taught in mindfulness-based interventions. There are a number of versions of the FB, but they all share the same goal: to teach the participants to focus their attention on the sensations of the breath and, in case it is compromised, to refocus on it with a quality of kindness (e.g., Kabat-Zinn, 1990; Segal et al., 2002a). The exercise encompasses two core elements of mindfulness: the awareness of the present moment, and the non-judgmental acceptance of one's moment-to-moment experiences (e.g., Baer, 2003; Kabat-Zinn, 1990). Previous studies have demonstrated that the ability to stay mindfully in contact with one's breathing negatively correlates with depression, rumination, and repetitive negative thinking (Burg \& Michalak, 2011). Moreover, it is positively related to enhanced levels of meta-awareness, nonattachment and mood, and less mind wandering (Levinson, Stoll, Kindy, Merry, \& Davidson, 2014). The engagement in this exercise results in brain activity alterations related to attentional processes (e.g., Bing-Canar, Pizzuto, \& Compton, 2016; Dickenson, Berkman, Arch, \& Lieberman, 2013).

Evidence of a single FB session on cognitive performance, however, is scarcer and shows non-conclusive results (for a systematic review, see Eisenbeck, Ruiz-Sanchez, Valdivia-Salas, \& Luciano, 2018). The possible effects of a single mindfulness exercise on cognitive functions among non-meditators may be important for several reasons. Controlled studies that implement short exercises may help us both to select and analyse the key practices involved in mindfulness-based therapies, as well as to understand how these therapies affect consciousness. With this understanding, it is possible to create more cost-effective short interventions and evaluate their utility in different contexts. For instance, there is only limited data available that a short mindfulness practice may help to enhance performance during a university exam (Ramsburg $\&$ Youmans, 2013) and facilitate the learning of new words (Bonamo, Legerski, \& Thomas, 2014). However, it is unknown whether a single FB session is effective for all types of cognitive performance and in all contexts that require concentration, or whether there are tasks and activities that could benefit more from such a short mindfulness practice. Controlled studies would be able to unfold these questions.

In controlled laboratory settings, there is some evidence that the FB can immediately enhance performance of the working memory and executive functioning tasks, such as the classical and emotional versions of the Stroop task (e.g., Lee \& Orsillo, 2014; Polak, 2009; Wenk-Sormaz, 2005) and the n-back test (e.g., Zeidan, Johnson, Diamond, David, \& Goolkasian, 2010). But most of the studies report the lack of effect on simple sustained attention tasks, such as the Eriksen Flanker Task (Larson, Steffen, \& Primosch, 2013) and the Attention Network Task (Polak, 2009). These tasks involve time pressure by measuring response latencies to detect stimuli on the screen in the presence of other distracting stimuli. To be more precise, the participants have to respond each time as quickly and accurately as possible to which direction an arrow is pointing in the presence of various disturbing stimuli. It is yet to be discovered whether a single session of FB may affect different types of simple sustained attention tasks, such as those in which the participants have to remain focused on a monotonous 
stimuli detection task that allows them to regulate their own pace of execution. These types of tasks are more similar to real-life situations, thus their testing would increase the ecological validity and the usefulness of the findings. Thus, the first aim of this study was to examine the effect of the FB on an attention task with the above features, namely the Concentrated Attention task of the Toulouse-Pierron Factorial Battery (Roffé, 2009).

With regard to cognitive tasks that measure memory processes, the evidence is mixed. Some of the previous experiments reported beneficial effects of a short FB practice for memory (Hammond, Wagstaff, \& Cole, 2006; Ramsburg \& Youmans, 2013; Wagstaff, Brunas, Wagstaff, Cole, \& Wheatcroft, 2004a; Wagstaff et al., 2004b; Wagstaff, Wheatcroft, Caddick, Kirby, \& Lamont, 2011) while other studies did not (Alberts \& Thewissen, 2011; Polak, 2009). These differences may be due to the different methodologies employed, including the features of the FB and the experimental tasks (for detailed analysis, see Eisenbeck et al., 2018). For instance, the studies that obtained unfavourable results of the FB protocol used lists of unrelated words (Alberts $\&$ Thewissen, 2011; Polak, 2009), whereas studies with favourable results used tasks with familiar or meaningful recall material, such as a university lecture (Ramsburg \& Youmans, 2013), human faces (Wagstaff et al., 2004a), an emotionally salient event (Wagstaff et al., 2004b), a crime videotape (Hammond et al., 2006), and a neutral videotape (Wagstaff et al., 2011). However, no study has shown this effect using more reliable, validated measures. In light of the possibility that the familiarity of the recall material may be relevant and that it may be sensitive to the effects of a short mindfulness practice, the second aim of the study was to employ a brief and validated memory task (Logical Memory Subtest I from the Wechsler Memory Scale III; Psychological Corporation, 1997) that uses familiar and meaningful recall material.

In addition to cognitive measures, previous studies assessed the participants' mood after the FB protocol. In these experiments, the FB was shown to significantly improve mood after negative mood induction and exposure to obsessive thoughts (e.g., Arch \& Craske, 2006; Broderick, 2005; Erisman \& Roemer, 2010; Hilt \& Pollak, 2012; Wahl, Huelle, Zurowski, \& Kordon, 2013). However, the FB did not seem to affect mood evaluations when the participants engaged in neutral attention and memory tasks (e.g., Alberts \& Thewissen, 2011; Ramsburg \& Youmans, 2013; Zeidan et al., 2010). None of the former cognitive studies measured mood across the entire experimental session (initial assessment, pretest, and posttest). This may be relevant, because monitoring the participants' mood during the whole experimental procedure provides information on how both the tasks and the different experimental conditions affect the participants' emotional state. Consequently, the third aim of this study was to assess mood evaluations during the entire experimental session with a validated mood scale, the Positive and Negative Affect Scale (PANAS; Watson, Clark, \& Tellegen, 1988).

Summarising, the data are inconclusive regarding whether or not a single FB session would improve cognitive performance and mood evaluations. We believe that such evidence could make an impact on designing evidence-based coping strategies to be readily used when confronted with a demanding situation, such as an exam or a job interview, and it would help to determine in which context short exercises are beneficial. In line with this, the present study focused on examining the effects of a short FB protocol on cognitive tasks and mood evaluations among non-meditator participants. It set three aims: (a) to assess the effects of the FB on a sustained attention task that does not involve constant time pressure, (b) to assess the effects of the FB 
on a memory task that implements meaningful recall material, and (c) to monitor the mood of the participants across the entire experimental session. For these purposes, the participants completed attention and memory tasks prior to and after practising a short FB (FB condition) or listening to an irrelevant audiorecording (Control condition). Mood was assessed across the whole experimental procedure: at initial assessment, pretest, and posttest. As mindfulness interventions are typically introduced in group format, the present study was also administered in this way.

\section{Method}

\section{Participants}

Undergraduate students were recruited through in-class announcements and were given course credits for their participation. A total number of 46 psychology students completed the experiment, including 27 females (59\%), with ages ranging between 20 and $52(M=25.24, S D=7.97)$. The participants were randomly assigned to two conditions: FB and Control. There was a total of 23 participants in the FB condition (14 females; age range: $20-52$ years; $M=25.91, S D=10.11$ ) and 23 in the Control condition ( 13 females; age range: $20-41$ years; $M=24.55, S D=5.01$ ). All participants were non-meditators and none of them had severe mental illness.

\section{Interventions}

Participants were randomly assigned to one of the two experimental conditions. In each condition, they listened to an audio recording containing either the FB (FB condition) or the control exercise (Control condition). Both recordings lasted 13 minutes and 11 seconds and were narrated by the same male voice.

Focused Breathing exercise (FB). A classic version of the FB was implemented (Segal, Williams, \& Teasdale, 2002b, pp. 172-173). This exercise has been used in a number of previous experiments (e.g., Arch \& Craske, 2006; Erisman \& Roemer, 2010; Feldman, Greeson, \& Senville, 2010; McHugh, Simpson, \& Reed, 2010; McHugh \& Wood, 2013). It consists of the following instructions: (a) close your eyes; (b) settle into a comfortable sitting position; (c) bring awareness to your body; (d) focus on the sensations of your breathing, and (e) focus your attention back to your breath in case it wanders with a posture of acceptance and curiosity for the present moment.

Control exercise. Similarly to previous studies, participants listened to the audiobook version of the first part of the first chapter of JRR Tolkien's The Hobbit (e.g., Kramer et al., 2013; Zeidan et al., 2010).

\section{Materials}

The effects of the protocols were assessed with measures of attention, memory, and mood. In order to assure the normality of the sample on mindfulness skills, data for trait mindfulness were also collected. All materials are available upon request.

Kentucky Inventory of Mindfulness Skills. The unpublished Spanish version of the Kentucky Inventory of Mindfulness Skills (KIMS; Baer, Smith, Hopkins, Krietemeyer, $\&$ Toney, 2006) was administered. The questionnaire contains 39 items measuring four different trait mindfulness skills: Observing (e.g., 'I pay attention to whether my muscles are tense or relaxed'), Describing (e.g., 'I'm good at finding the words to describe my feelings'), Acting with awareness (e.g., 'When I'm reading, I focus all my 
attention on what I'm reading') and Accepting without judgment (e.g., a reverse item: 'I disapprove of myself when I have irrational ideas'). Participants rate the extent to which each statement is true for them, by using a 5-point Likert scale ranging from 1 (never or very rarely true) to 5 (almost always or always true). Higher scores indicate higher mindfulness skills on each subscale. The Observing subscale ranges from 12 to 60; Describing from 8 to 40, Acting with awareness from 10 to 50, and Accepting without judgment from 9 to 45 . The KIMS is a valid instrument with good internal consistency; alpha coefficients for Observing, Describing, Acting with awareness and Accepting without judgment were .91, .84, .76, and .87 respectively (Baer et al., 2006). Cronbach's alphas in the present study for these subscales were $.82, .51, .74$, and .92 , respectively.

Attention test. A modified version of the Concentrated Attention task was used (from now on to be referred to as attention task), which is included in the ToulousePierron Factorial Battery (Roffé, 2009). This task assesses concentrated attention by calculating the number of correctly marked target figures among non-target figures in a limited period of time. The participants are given an A4 sized sheet of paper containing 1,200 small figures, arranged in 30 rows, with 40 figures per row. The figures consist of a square and a short line, which may extend out from the top, the bottom, or any of the four corners. The sheet contains a total number of six different variations of the figure - 200 of each. At the top of the sheet, three exemplars of the figures are shown that are deemed as targets. Targets (600 figures in total) and non-targets are randomly distributed on the sheet. The participants are instructed to correctly mark with a pen as many of the three target figures as they can. They are instructed to start the search at the upper left corner of the sheet and proceed row by row. In the original task, the completion time is 10 minutes, but in order to prevent possible ceiling effects, we set the completion time to 8 minutes.

We used two of the concentrated attention indexes included in the original test (Roffé, 2009): (a) Correct Responses, calculated by adding the number of correctly marked targets and correctly unmarked non-targets; and (b) Global Index, calculated by subtracting the number of errors (incorrectly unmarked targets and incorrectly marked non-targets) from the number of Correct Responses. Correct Responses range from 0 to 1,200 and Global Index ranges from -1,200 to 12,00.

Memory test. We employed a modified version of the Logical Memory Subtest I from the Wechsler Memory Scale III (from now on to be referred to as memory task; Psychological Corporation, 1997). This task consists of a series of short stories. All stories are equivalent in length (30 seconds), thematic unit score (general information included in the story; e.g., reference to the gender of the main character), and recall unit score (exact names and numbers included in the story; e.g., the name of the main character). During this task, participants are instructed to pay close attention to the story they are listening to and are asked to recall all the details for two minutes immediately after listening.

In this experiment, two stories were implemented: Story A (during pretest) and Story B (during posttest). Both were narrated by audio recordings using the same male voice. In order to reduce familiarity with the task, minor changes were introduced, that is, name of the main character of Story A and the number of children in Story B. Also, because of the group presentation format, the participants had to write down their answers. We used the official scoring sheet to assess participants' memory. Maximum scores were 7 points for thematic unit score and 25 points for recall unit score. 
Mood. The Spanish version of the PANAS (Sandín et al., 1999; Watson et al., 1988) was employed. The PANAS is a 20 -item scale containing 10 positive affect items (PANAS-P: active, alert, attentive, determined, enthusiastic, excited, inspired, interested, proud, and strong) and 10 negative affect items (PANAS-N: afraid, ashamed, distressed, guilty, hostile, irritable, jittery, nervous, scared, and upset). Participants rate their current feelings on a 5-point Likert scale ranging from 1 (very slightly or not at all) to 5 (extremely). Both PANAS-P and PANAS-N subscales range from 10 to 50 , with higher scores indicating higher affect (e.g., very enthusiastic or very hostile). Lately, researchers have been combining them to create a measure of 'hedonic balance' (e.g., Diehl, Hay, \& Berg, 2011; Mauss, Tamir, Anderson, \& Savino, 2011). The ratio of positive to negative affect was calculated for each participant by dividing the total number of positive effects by the total number of negative effects. The hedonic ratio appears to be a more sensitive indicator of mental health status, with a hedonic balance score of 2.9 or higher thus reflecting psychological wellbeing and flourishing (Diehl et al., 2011). The PANAS-P and PANAS-N subscales show high levels of internal consistency, convergent and discriminant validity, with Cronbach's alphas of $.89-.91$ (PANAS-N) and .87 - .89 (PANAS-P; Sandín et al., 1999). Reliability for our sample was as follows: .86 (initial assessment), .83 (pretest) and .85 (posttest) for PANAS-N, .86 (initial assessment), .92 (pretest) and .91 (posttest) for PANAS-P.

\section{Design and Procedure}

The design included a between-subjects variable (Condition: FB or Control) and three within-subject variables, namely: (a) mood, with measurements at three different times (initial assessment, pretest, and posttest); (b) attention; and (c) memory, with measurements at two different times (pretest and posttest). During the recruitment process, participants were only informed that they would participate in a study in which they would complete various tasks, questionnaires and a short exercise. The experiment took place in a regular university classroom and lasted approximately 50 minutes. They were randomly assigned to one of two conditions (FB and Control). In both conditions, the experimental protocols were implemented in group format, with seven to twelve participants per group. Three experimenters (one female, two males) who conducted the study read all instructions from a sheet of paper without any further contact with the participants.

Upon arrival, the participants received information about the duration of their participation and the activities they would perform (paper and pencil cognitive tasks, mood evaluations, and a short exercise). They were also informed that they could quit at any time during the procedures. The experiment started after participants signed the informed consent form.

During the initial assessment, participants completed the KIMS, PANAS-N and PANAS-P. They were also required to write down any previous or current diagnosis of mental disorder. At the end of this phase, the experimenter collected the questionnaires.

During the pretest, the participants were given sheets of paper containing the attention task, the PANAS-N and PANAS-P, all facing down, and a blank page to use during the memory task. First, they were presented with the memory task using Story A. They wrote down their answers in two minutes. The experimenters then collected the sheets and explained the attention task. Once the instructions had been understood, the experimenter asked the participants to turn the attention task sheet 
face up and start with the task. At the end of the completion period, the participants were instructed to hand the sheets over to the experimenter. To end this phase, they reported their current mood by filling out the PANAS-N and PANAS-P. Upon completion, the experimenters collected the questionnaires, leaving the desks empty for the next phase. Subsequently, participants in both conditions were informed that they would start a short exercise and were instructed to listen to a recording, preferably with their eyes closed. They either listened to the FB or Control audiotape, then immediately started the posttest.

During the posttest, memory, attention, and current mood were assessed, similarly to the pretest. However, for the memory task, participants were presented with Story B. As for the attention task, a different sheet was employed, with new targets and a different distribution of targets and non-targets.

Finally, the participants were requested to describe their meditation experience so as to select only non-meditators for the final analyses. First, they had to rate their experience with meditation and similar practices on a 5-point scale ranging from 1 (not at all) to 5 (a lot). After that, they had to describe what type of practices they were familiar with. Participants rating their previous experience as 3 or higher, and mentioning meditation or mindfulness as their previous practices were deemed as mediators. To end the procedures, the participants were fully debriefed.

\section{Data Reduction and Analytic Strategy}

A total number of five participants were eliminated from the final analysis, based on their performances on the memory task during pretest. The performance of three participants differed more than two standard deviations from the sample average, and two participants reported large amounts of unrelated information to Story A. All participants were deemed as non-meditators and all of them finished the experiment. Final analyses thus were conducted on the performance of 41 participants, 21 in the FB condition and 20 in the Control condition. Data from the attention task of two control participants and from mood evaluations of one FB participant were not analysed due to data loss.

To begin with, descriptive analyses were performed (frequencies, means, and standard deviations). In order to test for differences between the two conditions for demographic data and initial tendencies, independent sample $t$ tests were carried out on continuous variables (age, KIMS, PANAS-N, and PANAS-P) along with a chi-square test on gender. Further gender differences in respect of all outcome variables were analysed with independent samples $t$ tests and Mann-Whitney's U tests (whenever the assumptions of the $t$ test were not met). Differences based on age were assessed with the Pearson correlation coefficient for normally distributed variables and with Spearman's rank correlation when the variables were not normally distributed. Mann-Whitney's U tests were performed to assess possible differences between the experimenters in each condition.

In order to test for differences between conditions during pretest, independent samples $t$ tests and Mann-Whitney's $U$ tests (whenever the assumptions of the $t$ test were not met) were conducted for memory test performance (recall unit score and thematic unit score), attention test performance (Correct Responses and Global Index), and current mood (PANAS-N, PANAS-P and hedonic balance).

To evaluate the effect of the FB and Control protocols on attention and memory, we conducted four separate mixed analyses of variance (ANOVAs) for total Correct 
Responses, Global Index, recall unit score and thematic unit score. We used Test as a within-subject factor (pretest, posttest) and Condition as a between-subject factor (FB, Control). In case of a significant interaction effect, paired-samples $t$ tests for normally distributed variables were conducted in each condition.

Finally, three $3 \times 2$ (measuring time: initial assessment, pretest, posttest $\times$ Condition: FB, Control) mixed-design ANOVAs were performed on PANAS-N, PANAS$\mathrm{P}$, and hedonic balance to assess possible mood changes during the experimental session.

Tests of normality, sphericity, and homoscedasticity were carried out to ensure that the data met the assumptions of each test. As mixed ANOVA is a robust test for small violations of normality, it was implemented in each case (Glass, Peckham, \& Sanders, 1972). When necessary, the Greenhouse-Geisser degrees of freedom adjustment procedure was applied to correct for violations of sphericity. Cohen's $d$ effect sizes were calculated for $t$ tests and effect sizes of .02, .05, and .08 were considered small, medium, and large respectively (Cohen, 1988). Partial eta squared $\left(\eta^{2}\right)$ were calculated in order to determine effect sizes for ANOVAs. Effect sizes of .02, .13, and .26 were considered small, medium, and large respectively (Cohen, 1988). All tests were performed two-tailed and a significance of $p>.05$ was applied.

\section{Results}

Data regarding trait mindfulness and demographic variables will be presented first, followed by the results of attention, memory, and mood. In general, no differences were found between the experimenters during any of the assessments, $p>.05$.

\section{Trait Mindfulness and Demographic Variables}

Table 1 shows descriptive statistics for KIMS during the initial assessment and significance levels of the $t$ tests. All participants fell into normal reference ranges on KIMS (within two standard deviations from the normative data) and averages were similar to those obtained in normative samples (e.g., Baer et al., 2006). There were no significant differences between conditions in trait mindfulness, $p>.05$. Age and gender did not affect any of assessed variables of the study, with two exceptions: younger participants seemed to have higher recall unit score both at pretest, $r(39)=$ $-.41, p=.008$, and at posttest $r(39)=-.42, p=.007$, and male participants showed a tendency to report slightly better mood measured by PANAS-P during the initial assessment, $t(38)=2.05, p=.047, d=.65$.

\section{Attention Test}

Average data from the Concentrated Attention task of the Toulouse-Pierron Factorial Battery are shown in Table 2. At pretest, there was great variability among participants. Correct Responses ranged between 315 and 804 for the FB condition and between 266 and 753 for the Control condition, while the Global Index ranged between 287 and 716 for the FB condition and between 258 and 713 for the Control condition. Averages did not differ between conditions at pretest, $p>.05$ (see Table 2).

Posttest ranges were similar (Correct Responses: between 399 and 713 for FB and between 284 and 707 for Control; Global Index: between 368 and 651 for FB and between 278 and 678 for Control). However, most participants performed worse at posttest than at pretest (Correct Responses: 15 of 20 participants for FB and 14 of 21 


\section{TABLE 1}

Initial Assessment (Age, Gender, KIMS and PANAS)

\begin{tabular}{lccr}
\hline & FB $(n=20) M(S D)$ & Control $(n=21) M(S D)$ & $p$ \\
\hline Age & $26.65(10.67)$ & $24.38(5.19)$ & .388 \\
Women* & $11(57.89 \%)$ & $11(52.38 \%)$ & .871 \\
KIMS Total (39-195) & $134.65(14.83)$ & $139.47(19.62)$ & .382 \\
KIMS Observing (12-60) & $41.60(8.22)$ & $41.43(7.58)$ & .945 \\
KIMS Describing (8-40) & $28.75(5.69)$ & $33.48(11.5)$ & .106 \\
KIMS Awareness (10-50) & $32.90(5.01)$ & $32.57(4.96)$ & .834 \\
KIMS Acceptance (9-45) & $31.40(8.93)$ & $32.00(7.02)$ & .812 \\
PANAS-N** (10-50) & $16.26(6.67)$ & $15.05(4.80)$ & .509 \\
PANAS-P** (10-50) & $28.00(6.42)$ & $30.71(8.06)$ & .250 \\
Hedonic balance** (-4-4) & $1.17(.82)$ & $1.57(.84)$ & .142 \\
\hline
\end{tabular}

Note: $\mathrm{FB}=$ Focused Breathing exercise condition; Control = control condition; KIMS = Kentucky Inventory of Mindfulness Skills; PANAS-N = Negative affect subscale of the Positive and Negative Affect Scale; PANAS-P = Positive affect subscale of the Positive and Negative Affect Scale; Hedonic balance $=$ the ratio of PANAS-P and PANAS-N.

* In the case of gender, frequency (and percentage) is reported.

**FB: $N=19$.

$p$ level $=$ significance of the independent $t$ tests between FB and Control.

\section{TABLE 2}

Pre-Post Data for Attention, Memory and Mood

\begin{tabular}{llrcc}
\hline & & & Pretest $M(S D)$ & Posttest $M(S D)$ \\
\hline Attention & Correct Responses & FB $(n=20)$ & $584.70(118.28)$ & $544.35(93.45)$ \\
task & $(0-980)$ & Control $(n=19)$ & $592.68(114.37)$ & $539.53(107.93)$ \\
& Global Index & FB $(n=20)$ & $536.40(105.39)$ & $508.90(87.53)$ \\
& $(-980-980)$ & Control $(n=19)$ & $552.89(105.39)$ & $511.84(104.09)$ \\
Memory & Information units & FB $(n=21)$ & $12.30(3.20)$ & $14.40(3.30)$ \\
task & $(0-25)$ & Control $(n=21)$ & $13.71(2.17)$ & $13.76(2.36)$ \\
& Information themes & FB $(n=20)$ & $5.25(1.16)$ & $5.90(1.00)$ \\
& (0-7) & Control $(n=21)$ & $5.55(1.05)$ & $5.52(.93)$ \\
Mood & PANAS-N & FB $(n=19)$ & $15.58(5.37)$ & $14.42(5.34)$ \\
& $(10-50)$ & Control $(n=21)$ & $14.24(4.65)$ & $14.43(5.40)$ \\
& PANAS-P & FB $(n=19)$ & $25.73(8.51)$ & $26.16(7.83)$ \\
& (10-50) & Control $(n=21)$ & $30.00(8.20)$ & $28.00(8.45)$ \\
& Hedonic balance & FB $(n=19)$ & $1.02(1.049$ & $1.17(.79)$ \\
& (-4-4) & Control $(n=21)$ & $1.58(.90)$ & $1.36(1.02)$ \\
\hline
\end{tabular}

Note: FB = Focused Breathing exercise condition; Control = Control condition; Attention task $=$ Concentrated Attention task of the Toulouse-Pierron Factorial Battery; Memory task = Logical Memory Subtest I from the Wechsler Memory Scale III; PANAS-N = Negative affect subscale of the Positive and Negative Affect Scale; PANAS-P = Positive affect subscale of the Positive and Negative Affect Scale; Hedonic balance $=$ The ratio of PANAS-P and PANAS-N. 
for Control; Global Index: 15 of 20 participants for FB and 15 of 21 for Control). For averages, see Table 2 .

When comparing the average performance as a function of Condition and Time, mixed ANOVAs revealed a main effect of Time for both attention indexes, that is, Correct Responses, $F(1,37)=23.73, p<.001, \eta^{2}=.391$, and Global Index, $F 1$, $37)=14.37, p=.001, \eta^{2}=.280$. No other effects were significant, $p>.05$. These data suggest that performance on the attention task declined at posttest, although it was not specific to the experimental condition.

\section{Memory Test}

Average data from the Logical Memory Subtest I from the Wechsler Memory Scale III are shown in Table 2. At pretest, participants showed relatively high performances when measured by the thematic unit score (range: between 4 and 7 for both conditions) and medium performances when measured by recall unit score (range: between 4 and 7 for FB and between 3 and 7 for Control). Pretest scores did not differ between conditions, $p>.05$ (see Table 2).

At posttest, thematic unit scores ranged between 4 and 7 for FB and between 3 and 7 for Control. The recall unit score ranged between 8 and 20 for FB and between 7 and 18 for Control. A higher number of participants enhanced their performances on FB as compared to Control (thematic unit score: 10 of 20 participants for FB and five of 21 for Control; recall unit score: 14 of 20 for FB and 10 of 21 for Control).

The condition $\times$ time ANOVA conducted on thematic unit score revealed a trend towards a significant interaction effect, $F(1,39)=4.10, p=.050, \eta^{2}=.095$. No other effects were significant, $p>.05$. This suggests that the participants did not perform better at posttest than at pretest. None of the conditions showed significant pre-post changes on the thematic unit score, $p>.05$.

The statistical comparison of the recall unit score as a function of Condition and Time revealed a main effect of Time, $F(1,39)=9.56, p=.004, \eta^{2}=.197$, and a significant interaction effect, $F(1,39)=8.73, p=.005, \eta^{2}=.183$. The participants in the FB condition showed a significant increase in information units from pretest to posttest, $t(19)=-5.05 p<.001, d=1.13$. Control participants did not show pre-post changes, $p>.05$, indicating that only the FB protocol was successful in enhancing the performance on the recall unit score.

To summarise, the FB protocol, as compared to Control, was successful in increasing the participants' performance for the recall unit score, but not for the thematic unit score.

\section{Mood}

Tables 1 and 2 show average data for PANAS-N, PANAS-P, and hedonic balance at initial assessment, pretest, and posttest. Table 1 also shows descriptive statistics for the initial assessment and significance levels of the $t$ tests. Participants in both the FB and Control conditions started the experiment in a neutral mood, measured both by PANAS-N (ranges: between 10 and 32 for FB; between 10 and 27 for Control) and PANAS-P (ranges: between 12 and 37 for FB; between 16 and 48 for Control). There were no significant differences between the conditions for any of the mood scales, $p>.05$ (see Table 1).

At pretest, participants' mood was generally neutral (PANAS-N range: between 10 and 27 for FB and between 10 and 27 for Control; PANAS-P range: between 12 
and 40 for FB and between 13 and 45 for Control). At posttest, the data were very similar, both for negative (range: between 10 and 26 for FB and between 10 and 27 for Control) and positive mood (range: between 13 and 42 for FB and between 11 and 44 for Control).

When averaged within conditions, the 3 (Time: initial assessment, pretest, posttest) $\times 2$ (Condition: FB, Control) mixed ANOVAs for PANAS-N, PANAS-P and hedonic balance showed neither significant interactions nor main effects, $p>.05$. Although the mean values of the hedonic balance show different tendencies in the two groups between the pretest and posttest (see Table 2), the analyses suggest that neither the tests nor the conditions changed significantly the participants' mood.

\section{Discussion}

The present study has analysed the effects of a short mindfulness protocol among nonmeditator undergraduate students with average levels of trait mindfulness skills. The experiment set three aims: (a) analysing the FB's effects on a monotonous sustained attention task without constant time pressure, (b) analysing the FB's effects on a validated memory task implementing meaningful and familiar recall material, and (c) monitoring the participants' mood across the entire experiment. Results showed no significant differences between the FB and Control conditions on the attention task and mood, but the FB, as opposed to Control, significantly increased performance on the memory task in terms of recall unit score reported.

At the initial measurement, male students showed slightly higher levels of positive mood, but gender did not affect any other variables in the study. Age exclusively affected the recall unit score, both at pretest and posttest. This effect may be due to the low sample size and the heterogeneity of age in the sample (there were a couple of participants over 35 years old), which may reflect different characteristics of the participants, such as different cognitive and motivational levels.

Regarding the attention task, the FB was not effective at increasing the participants' performance. These results are similar to those obtained in previous studies (Larson et al., 2013; Polak, 2009). However, in this experiment, most participants actually decreased their performances at posttest. One possible explanation for this effect may stem from the characteristics of the task that are more similar to real-life situations, rather than those used in previous studies. In the experiments of Larson and colleagues (2013) and Polak (2009), computerised stimulus detection tasks were employed (the Eriksen Flanker Task and the Attention Network Task respectively). In our study, however, participants regulated their own pace of execution because response latency was not our target, and this may have rendered the task as monotonous and boring. As such, it may have been relatively easy for the participants to disconnect from the task, and a single session of FB may not be enough to prevent declines due to the unappealing characteristics of the attention task. Interestingly, even studies focusing on the effects of long-term mindfulness practices reported mixed results with regard to whether they were beneficial for attentional abilities or not (e.g., Jha, Stanley, Kiyonaga, Wong, \& Gelfand, 2010; Lykins, Baer, \& Gottlob, 2012; Moore, Gruber, Derose, \& Malinowski, 2012; Valentine \& Sweet, 1999). All in all, the present findings suggest the likely insensitivity of simple and monotonous attention tasks to short mindfulness interventions, in accordance with some previous considerations (e.g., Chiesa et al., 2011; Eisenbeck et al., 2018; Gallant, 2016). 
In contrast, significant differences were observed between the FB and Control conditions for the memory task in terms of recall unit score (specific information about the stories; e.g., the main character had three children), but not for the thematic unit score (general information; e.g., the main character was a mother). The lack of significant effects in this part of the task may be due to the generally high performances at pretest that possibly produced ceiling effects. This explanation is supported by the data showing that on average, participants in both conditions improved their performances with the recall unit score but not the thematic unit score. These differences are similar to previous findings, especially those in which the participants had to recall familiar, meaningful material and remember connections between elements, such as a university lecture, remembering human faces, or the contents of short video clips (e.g., Hammond et al., 2006; Ramsburg \& Youmans, 2013; Wagstaff et al., 2004a; Wagstaff et al., 2004b; Wagstaff et al., 2011). This experiment added evidence to the memory-enhancing effects of the FB and raised the possibility that it can be especially useful for somewhat meaningful tasks in which it may be important to remember connections between elements. However, the present study had higher experimental control compared to previous experiments, as a pre-post design and a shorter, validated task were used.

As for the mood evaluations, non-significant differences were observed between the two conditions. Previous studies showed similar results when a short FB was implemented and the participants engaged in non-stressful cognitive tasks (e.g., Alberts \& Thewissen, 2011; Polak, 2009; Ramsburg \& Youmans, 2013; Zeidan et al., 2010). Nevertheless, the present study was the first to monitor mood across the entire experimental session (initial assessment, pretest, posttest) while engaging in neutral cognitive tasks. It allowed us to observe that the participants started the experimental session in a relatively neutral mood and subsequently, neither the experimental tasks nor the interventions induced significant changes in the hedonic balance of the participants. Our study confirmed that a single FB protocol does not change neutral mood, and thus its memory performance-improving effects cannot be mediated simply through mood changes (for similar considerations, see Alberts \& Thewissen, 2011; Ramsburg \& Youmans, 2013; Zeidan et al., 2010).

Overall, one of the most relevant contributions of this experiment was the different effects observed on the attention and memory tasks without effects on mood, suggesting that task characteristics may have played an important role. There were previous studies that had similar theoretical considerations arguing that a short mindfulness exercise may enhance memory performance but not simple attentional abilities (e.g., Chiesa et al., 2011; Eisenbeck et al., 2018; Gallant, 2016; Lykins et al., 2012). However, this was the first empirical study that confirmed these differences in a single experiment, using validated measures. It is feasible to argue that such a short mindfulness practice may not affect simple attentional abilities and may not have been potent enough to counterbalance the unattractive characteristics of the task. This task may not have been complex and appetitive enough to capture possible behavioural changes induced by a brief FB protocol. In contrast, the memory task may have required higher levels of attention for a shorter period of time, as well as establishing relations between events in order to remember the story. This task might have been also more familiar, attractive and entertaining than the attention task, being somehow more meaningful for the participants to remember a story with names and places than to detect differences between abstract figures without any experimentally provided value. Thus, this task may have been more suitable to capture the initial effects of a short mindfulness 
practice. These findings highlight the possibility that the practice of a short FB session may not be beneficial in all contexts.

Besides the promising results, a number of limitations must be noted. Our participants were a relatively small sample of undergraduate students; therefore the results should not be generalised to other populations. No manipulation checks were employed in the study. For instance, no state mindfulness questionnaire, such as the Mindful Attention Awareness - State Version (MAAS-S; Brown \& Ryan, 2003) was used, which could have provided information on the impact of the FB on selfreported mindfulness levels. As such, it is unknown whether the FB participants actually engaged in the exercise. Moreover, there is no information about whether participants in either condition believed that the exercise would help them to enhance their performance during posttest. Future studies should include such manipulation checks in order to precisely monitor the participants' behaviour during the experiment and to provide more detailed analyses; for instance, to only assess those participants who engaged in the mindfulness practice.

There was a possible ceiling effect in the thematic unit score of the memory task that may have clouded our results. No measurement was implemented for individual preferences, previous practice of the tasks, or preferences towards the FB. This may have affected the obtained results, because some participants may have preferred one of the tasks or conditions more than the others, creating differences in their levels of intrinsic motivation to perform better.

We used the FB protocol, thus our conclusions cannot be extrapolated to other mindfulness exercises. Mindfulness meditation encompasses a family of different techniques (e.g., Kabat-Zinn, 1994; Segal et al., 2002a) that can generally be divided into two broad categories: focused attention exercises, such as the FB, when the practitioners focus their attention on a chosen object; and open monitoring exercises that are non-reactive monitoring practices of the current experience (Lutz, Slagter, Dunne, \& Davidson, 2008). Open monitoring exercises, such as the body scan exercise or the mindful raisin-eating exercise (e.g., Kabat-Zinn, 1990, 2003), have shown different behavioural outcomes when compared to the FB (e.g., Colgan, Christopher, Michael, \& Wahbeh, 2015; Colzato, Ozturk, \& Hommel, 2012; Feldman et al., 2010; Valentine \& Sweet, 1999). For example, a short body scan exercise improved attentional control and the ability to distinguish between signal and noise, as it reduced tactile misinterpretation and increased sensitivity on a somatic signal detection task (Mirams, Poliakoff, Brown, \& Lloyd, 2013). Future studies should emphasise the characteristics of the implemented mindfulness exercises and take into account the possibility that other practices may have different effects on the participants' performance.

Alternative explanations of the findings are also plausible. The discrepancies between the two experimental tasks are numerous. Neither a 'meaningless' memory task, nor a more appetitive sustained attention task were implemented in this experiment, 66 thus it is unknown whether these characteristics could have played an important role. Moreover, in this study, the memory task was presented first, followed by the attention task; therefore, sequential effects may have had an impact on the results. It is also unknown whether similar results might have been obtained with cognitive tasks that induce stress or negative affect. Future research should assess tasks that differ only in one dimension (e.g., level of cognitive load or meaningfulness). It may also be useful to compare the FB with different control conditions and assess its effects on different populations in order to fully understand the processes involved in mindful change. 
Besides the limitations, the present study has shown high ecological validity for interventions that are administered in group format among college students, and the findings show relevant clinical implications. It has been confirmed that a brief FB can be easily administered among non-meditator university students and that even such a short practice can help to improve performance on more complicated, familiar and meaningful tasks, such as an exam. However, it may not be the best option to enhance performance on monotonous sustained attention tasks. These findings support the utility of practising a short mindfulness exercise before a task that requires complex cognitive functioning, such as an exam, but this practice is most probably not as useful before tasks that only require elevated attention levels.

In summary, this was the first experiment showing that the $\mathrm{FB}$, even after a very brief practice period, improved cognitive performance for a meaningful memory task, while no relevant changes were observed for a monotonous attention task. Moreover, these effects were not mediated by mood changes. This study's explanation of the results, based on the task characteristics, could open a new line of research focusing on the interplay between the type of task - as an analogue of life situations and the mindfulness exercise. The profound understanding of the situations in which mindfulness may be beneficial and in those that it is not can help us to develop more effective practices in the future.

\section{Funding}

This study was funded by the Ministry of Education, Culture and Sport of Spain (grant number: AP-2009-1232).

\section{Conflict of Interest}

All authors declare that they have no conflict of interest.

\section{Ethical Approval}

All procedures performed in studies involving human participants were in accordance with the ethical standards of the institutional and/or national research committee and with the 1964 Helsinki declaration and its later amendments or comparable ethical standards.

\section{Informed Consent}

Informed consent was obtained from all individual participants included in the study.

\section{References}

Alberts, H.J., \& Thewissen, R. (2011). The effect of a brief mindfulness intervention on memory for positively and negatively valenced stimuli. Mindfulness, 2, 73-77. doi:10.1007/s12671-011-0044-7

Arch, J.J., \& Craske, M.G. (2006). Mechanisms of mindfulness: Emotion regulation following a focused breathing induction. Behaviour Research and Therapy, 44, 1849-1858. doi:10.1016/j.brat.2005.12.007

Baer, R.A. (2003). Mindfulness training as a clinical intervention: A conceptual and empirical review. Clinical Psychology: Science and Practice, 10, 125-143. doi:10.1093/clipsy.bpg015

Baer, R.A., Smith, G.T., Hopkins, J., Krietemeyer, J., \& Toney, L. (2006). Using self-report assessment methods to explore facets of mindfulness. Assessment, 13, 27-45. doi:10.1177/1073191105283504 
Bing-Canar, H., Pizzuto, J., \& Compton, R.J. (2016). Mindfulness-of-breathing exercise modulates EEG alpha activity during cognitive performance. Psychophysiology, 53, 1366-1376. doi:10.1111/psyp. 12678

Bonamo, K., Legerski, J.-P., \& Thomas, K. (2014). The influence of a brief mindfulness exercise on encoding of novel words in female college students. Mindfulness, 6, 535-544. doi:10.1007/s12671-014-0285-3

Broderick, P.C. (2005). Mindfulness and coping with dysphoric mood: Contrasts with rumination and distraction. Cognitive Therapy and Research, 29, 501-510.

Brown, K.W., \& Ryan, R.M. (2003). The benefits of being present: Mindfulness and its role in psychological well-being. Journal of Personality and Social Psychology, 84, 822-848.

Brown, K.W., Ryan, R.M., \& Creswell, J.D. (2007). Mindfulness: Theoretical foundations and evidence for its salutary effects. Psychological Inquiry, 18, 211-237.

Burg, J., \& Michalak, J. (2011). The healthy quality of mindful breathing: Associations with rumination and depression. Cognitive Therapy and Research, 35, 179-185. doi:10.1007/s10608-010-9343-x

Chambers, R., Lo, B., \& Allen, N. (2008). The impact of intensive mindfulness training on attentional control, cognitive style, and affect. Cognitive Therapy and Research, 32, 303-322. doi:10.1007/s10608-007-9119-0

Chiesa, A., Calati, R., \& Serretti, A. (2011). Does mindfulness training improve cognitive abilities? A systematic review of neuropsychological findings. Clinical Psychology Review, 31, 449-464. doi:10.1016/j.cpr.2010.11.003

Cohen, J. (1988). Statistical power analysis for the behavioral sciences. Hillsdale, NJ: Lawrence Erlbaum.

Colgan, D.D., Christopher, M., Michael, P., \& Wahbeh, H. (2015). The body scan and mindful breathing among veterans with PTSD: Type of intervention moderates the relationship between changes in mindfulness and post-treatment depression. Mindfulness, 7, 372-383. doi: $10.1007 / \mathrm{s} 12671-015-0453-0$

Colzato, L.S., Ozturk, A., \& Hommel, B. (2012). Meditate to create: The impact of focused-attention and open-monitoring training on convergent and divergent thinking. Frontiers in Psychology, 3 , 116

Dickenson, J., Berkman, E.T., Arch, J., \& Lieberman, M.D. (2013). Neural correlates of focused attention during a brief mindfulness induction. Social Cognitive and Affective Neuroscience, 8 , 40-47. doi:10.1093/scan/nss030

Diehl, M., Hay, E.L., \& Berg, K.M. (2011). The ratio between positive and negative affect and flourishing mental health across adulthood. Aging 8 Mental Health, 15, 882-893. doi: $10.1080 / 13607863.2011 .569488$

Eisenbeck, N., Ruiz-Sanchez, L.J., Valdivia-Salas, S. \& Luciano, C. (2018). Does mindfulness immediately affect cognitive performance among non-meditators? A systematic review of the focused breathing exercise on cognitive measures in controlled settings. Manuscript submitted for publication.

Erisman, S.M., \& Roemer, L. (2010). A preliminary investigation of the effects of experimentally induced mindfulness on emotional responding to film clips. Emotion, 10, 72-82. doi: $10.1037 / \mathrm{a} 0017162$

Feldman, G., Greeson, J., \& Senville, J. (2010). Differential effects of mindful breathing, progressive muscle relaxation, and loving-kindness meditation on decentering and negative reactions to repetitive thoughts. Behaviour Research and Therapy, 48, 1002-1011. doi:10.1016/j.brat.2010.06.006

Gallant, S.N. (2016). Mindfulness meditation practice and executive functioning: Breaking down the benefit. Consciousness and Cognition, 40, 116-130. doi:10.1016/j.concog.2016.01.005

Glass, G.V., Peckham, P.D., \& Sanders, J.R. (1972). Consequences of failure to meet assumptions underlying the fixed effects analyses of variance and covariance. Review of Educational Research, 42, 237-288.

Hammond, L., Wagstaff, G.F., \& Cole, J. (2006). Facilitating eyewitness memory in adults and children with context reinstatement and focused meditation. Journal of Investigative Psychology and Offender Profiling, 3, 117-130. 
Hilt, L.M., \& Pollak, S.D. (2012). Getting out of rumination: Comparison of three brief interventions in a sample of youth. Journal of Abnormal Child Psychology, 40, 1157-1165. doi:10.1007/s10802-012-9638-3

Jha, A.P., Stanley, E.A., Kiyonaga, A., Wong, L., \& Gelfand, L. (2010). Examining the protective effects of mindfulness training on working memory capacity and affective experience. Emotion, 10, 54-64. doi:10.1037/a0018438

Kabat-Zinn, J. (1990). Full catastrophe living: Using the wisdom of your body and mind to face stress, pain, and illness. New York, NY: Delta Trade Paperbacks.

Kabat-Zinn, J. (1994). Wherever you go, there you are: Mindfulness meditation in everyday life. New York, NY: Hyperion.

Kabat-Zinn, J. (2003). Mindfulness-based interventions in context: Past, present, and future. Clinical Psychology: Science and Practice, 10, 144-156. doi:10.1093/clipsy.bpg016

Keng, S.-L., Smoski, M.J., \& Robins, C.J. (2011). Effects of mindfulness on psychological health: A review of empirical studies. Clinical Psychology Review, 31, 1041-1056. doi: http://dx.doi.org/10.1016/j.cpr.2011.04.006

Khoury, B., Lecomte, T., Fortin, G., Masse, M., Therien, P., Bouchard, V., ... Hofmann, S.G. (2013). Mindfulness-based therapy: A comprehensive meta-analysis. Clinical Psychology Review, 33, 763-771.

Kramer, R.S., Weger, U.W., \& Sharma, D. (2013). The effect of mindfulness meditation on time perception. Consciousness and Cognition, 22, 846-852. doi:10.1016/j.concog.2013.05.008

Larson, M.J., Steffen, P.R., \& Primosch, M. (2013). The impact of a brief mindfulness meditation intervention on cognitive control and error-related performance monitoring. Frontiers in Human Neuroscience, 7, 308. doi:10.3389/fnhum.2013.00308

Lee, J.K., \& Orsillo, S.M. (2014). Investigating cognitive flexibility as a potential mechanism of mindfulness in generalized anxiety disorder. Journal of Behavior Therapy and Experimental Psychiatry, 45, 208-216. doi:10.1016/j.jbtep.2013.10.008

Levinson, D.B., Stoll, E.L., Kindy, S.D., Merry, H.L., \& Davidson, R.J. (2014). A mind you can count on: Validating breath counting as a behavioral measure of mindfulness. Frontiers in Psychology, 5, 1202. doi:10.3389/fpsyg.2014.01202

Lutz, A., Slagter, H.A., Dunne, J.D., \& Davidson, R.J. (2008). Attention regulation and monitoring in meditation. Trends in Cognitive Sciences, 12, 163-169. doi:10.1016/j.tics.2008.01.005

Lykins, E.L., \& Baer, R.A. (2009). Psychological functioning in a sample of long-term practitioners of mindfulness meditation. Journal of Cognitive Psychotherapy, 23, 226-241.

Lykins, E.L.B., Baer, R.A., \& Gottlob, L.R. (2012). Performance-based tests of attention and memory in long-term mindfulness meditators and demographically matched nonmeditators. Cognitive Therapy and Research, 36, 103-114. doi:10.1007/s10608-010-9318-y

Mauss, I.B., Tamir, M., Anderson, C.L., \& Savino, N.S. (2011). Can seeking happiness make people unhappy Paradoxical effects of valuing happiness. Emotion, 11, 807-815. doi:10.1037/a0022010

McHugh, L., Simpson, A., \& Reed, P. (2010). Mindfulness as a potential intervention for stimulus over-selectivity in older adults. Research in Developmental Disabilities, 31, 178-184. doi: http://dx.doi.org/10.1016/j.ridd.2009.08.009

McHugh, L., \& Wood, R. (2013). Stimulus over-selectivity in temporal brain injury: Mindfulness as a potential intervention. Brain Injury, 27, 1595-1599. doi:10.3109/02699052.2013.834379

Mirams, L., Poliakoff, E., Brown, R.J., \& Lloyd, D.M. (2013). Brief body-scan meditation practice improves somatosensory perceptual decision making. Consciousness and Cognition, 22, 348-359. doi:10.1016/j.concog.2012.07.009

Moore, A., Gruber, T., Derose, J., \& Malinowski, P. (2012). Regular, brief mindfulness meditation practice improves electrophysiological markers of attentional control. Frontiers in Human Neuroscience, 6, 18. doi:10.3389/fnhum.2012.00018

Polak, E.L. (2009). Impact of two sessions of mindfulness training on attention (Doctoral dissertation, Miami University). Retrieved from http://citeseerx.ist.psu.edu/viewdoc/ download?doi=10.1.1.469.7226\&rep=rep1\&type=pdf 
Psychological Corporation. (1997). The WAIS III - WMS III technical manual. San Antonio, TX: Author.

Ramsburg, J., \& Youmans, R. (2013). Meditation in the higher-education classroom: Meditation training improves student knowledge retention during lectures. Mindfulness, 5, 431-441. doi: $10.1007 / \mathrm{s} 12671-013-0199-5$

Roffé, M. (2009). Evaluación psicodeportológica. 30 test psicométricos y proyectivos. Buenos Aires, Argentina: Lugar Editorial.

Sandín, B., Chorot, P., Lostao, L., Joiner, T.E., Santed, M.A., \& Valiente, R.M. (1999). Escalas panas de afecto positivo y negativo: validación factorial y convergencia transcultural. Psicothema, $11,37-51$.

Segal, Z.V., Williams, J.M.G., \& Teasdale, J.D. (2002a). Mindfulness-based cognitive therapy for depression: A new approach to preventing relapse. New York, NY: Guilford Press.

Segal, Z.V., Williams, J.M., \& Teasdale, J.D. (2002b). Terapia cognitiva de la depresión basada en la consciencia plena. Un nuevo abordaje para la prevención de las recaídas. Bilbao, Spain: Desclée de Brouwer.

Shapiro, S.L., Carlson, L.E., Astin, J.A., \& Freedman, B. (2006). Mechanisms of mindfulness. Journal of Clinical Psychology, 62, 373-386. doi:10.1002/jclp.20237

Shonin, E., Van Gordon, W., \& Griffiths, M. (2013). Mindfulness-based interventions: Towards mindful clinical integration. Frontiers in Psychology, 4, 194.

Valentine, E.R., \& Sweet, P.L. (1999). Meditation and attention: A comparison of the effects of concentrative and mindfulness meditation on sustained attention. Mental Health, Religion $\mathfrak{E}$ Culture, 2, 59-70.

Wagstaff, G., Brunas-Wagstaff, J., Cole, J., \& Wheatcroft, J. (2004a). New directions in forensic hypnosis: Facilitating memory with a focused meditation technique. Contemporary Hypnosis, 21, $14-27$.

Wagstaff, G.F., Brunas-Wagstaff, J., Cole, J., Knapton, L., Winterbottom, J., Crean, V., \& Wheatcroft, J. (2004b). Facilitating memory with hypnosis, focused meditation, and eye closure. International Journal of Clinical and Experimental Hypnosis, 52, 434-455. doi:10.1080/00207140490889062

Wagstaff, G.F., Wheatcroft, J.M., Caddick, A.M., Kirby, L.J., \& Lamont, E. (2011). Enhancing witness memory with techniques derived from hypnotic investigative interviewing: Focused meditation, eye-closure, and context reinstatement. International Journal of Clinical and Experimental Hypnosis, 59, 146-164. doi:10.1080/00207144.2011.546180

Wahl, K., Huelle, J., Zurowski, B., \& Kordon, A. (2013). Managing obsessive thoughts during brief exposure: An experimental study comparing mindfulness-based strategies and distraction in obsessive-compulsive disorder. Cognitive Therapy and Research, 37, 752-761. doi: $10.1007 / \mathrm{s} 10608-012-9503-2$

Watson, D., Clark, L.A., \& Tellegen, A. (1988). Development and validation of brief measures of positive and negative affect: The PANAS scales. Journal of Personality and Social Psychology, 54, 1063-1070.

Wenk-Sormaz, H. (2005). Meditation can reduce habitual responding. Advances in Mind-Body Medicine, 21, 33-49.

Zeidan, F., Johnson, S.K., Diamond, B.J., David, Z., \& Goolkasian, P. (2010). Mindfulness meditation improves cognition: Evidence of brief mental training. Consciousness and Cognition, 19, 597-605. doi: $10.1016 / j$.concog.2010.03.014 